\title{
Large-Scale Hydrodynamic Simulations of Astrophysical Jets
}

\section{J.H. Beall, ${ }^{a, b, *}$ D.V. Rose, ${ }^{c}$ Kevin Lind, ${ }^{c}$ Michael T. Wolff, ${ }^{b}$ Brian van Soelen, ${ }^{d}$ Izak van der Westhuizen ${ }^{d}$ and Pieter Meintjes ${ }^{d}$}

${ }^{a}$ St. John's College, Annapolis, MD, USA

${ }^{b}$ Space Sciences Division, Naval Research Laboratory, Washington, DC, USA

${ }^{c}$ Voss Scientific, Albuquerque, NM, USA

${ }^{d}$ Department of Physics, University of the Free State, Bloemfontein, SA

E-mail: beall@sjc.edu

\begin{abstract}
We present recent results for fully three-dimensional (3-D) relativistic hydrodynamic (RHD), and relativistic magneto-hydrodynamic (RMHD) simulations of astrophysical jets. These efforts use the PLUTO code (Mignone et al. 2007) run in a highly parallelized environment. Here, we focus on especially long term simulations of the jet evolution. This work is an extension of simulations reported earlier (Beall et al., 2018), and shows the total time evolution of the RMHD jet increased by a factor of two, or roughly $250,000 \mathrm{cpu}$ hours of computer time and a total of 50 terabytes of data. This extends the previous work from 125,000 cpu hours and 25 terabytes of data, and provides an important test of the stability of the solvers used in our simulations. We also show an estimation of the $15 \mathrm{GHz}$ radio flux based on our RHD simulations post-processed to show the fully 3-dimensional radio-emission from the astrophysical jets. We do this in order to guide us in our expectations for the possible separate locations of the radio and $\gamma$-ray emission locations for a jet. It has not escaped our notice that this analysis naturally explains the apparent separation between radio and $\gamma$-ray emission in some astrophysical sources.
\end{abstract}

Keywords: jets, active galaxies, blazars, intracluster medium, non-linear dynamics, plasma astrophysics, computational fluid dynamics, relativistic, magnetized fluid flows.

Multifrequency Behaviour of High Energy Cosmic Sources - XIII - MULTIF2019

3-8 June 2019

Palermo, Italy

${ }^{*}$ Speaker 


\section{Introduction}

The current, high-resolution observations of astrophysical jets from VLBA (Very Long Baseline Arrays) and the Event Horizon Telescope Millimeter-Wave Interferometer Array (that is, the EHT, and CARMA: see, e.g., Lister et al. 2009), Beall (2015), and Lin et al. 2019, 2020) reveal complex structures in the cores of AGNs and microquasars, apparently caused by ejecta from the central engine. In fact, because of the recent observations using the millimeter-wave interferometer systems, including ALMA and CARMA), it has becoming possible to characterize these structures to scales of the black hole accretion disks in AGNs. The temporal and spatial structures of the jets at both microquasar and quasar scales (i.e., from sub-parsec to kiloparsecs scales) now show that the effects might be produced by quasi-periodic ejection processes in accretion disks.

The ejected material then interacts with the surrounding interstellar material in the source region. In the cases of AGN, this includes the Broad-Line Region (BLR) and Narrow-Line Region (NLR) clouds, and with ejecta from prior episodes of activity. A particularly interesting example of these complex interactions is shown by the galactic microquasar, Sco X-1 (Fomalhaut, Geldzahler, and Bradshaw, 2001).

Such observations can be used to delimit models of the jet-ambient-medium interactions. Based on an analysis of these data, we posit that a significant part of the observed phenomena comes from the interaction of the ejected material in flares with prior ejecta as well as interstellar material. Simulations of these phenomena clearly require Relativistic Magneto-Hydrodynamic (RMHD) modeling, since there is now clear evidence of significant magnetic fields on all jet scales.

\section{Scales of Jet Interactions with the Ambient Medium}

In this discussion, we focus on the large-scale, relativistic, magneto-hydrodynamic simulations which can be used to illuminate a number of interesting consequences of the jet's interaction with the ambient medium through which it propagates. These include acceleration and entrainment of the ambient medium, the effects of shock structures on star formation rates, and other effects originating from ram pressure and turbulence generated by the jet (see, e.g., Basson and Alexander, 2002; Zanni et al. 2005; and Krause and Camenzind, 2003; Perucho et al., 2010; Perucho (2012); Belyaev, et al., 2019; and Mukherjee, D., et al., 2018).

The term "Large-scale" with respect to simulations discussed herein has two different faces: first, the large number of cells in 3-D simulations, and second, the long time-scale allowed for the accumulation of data from the simulations in order to show the manner in which the jets evolve in their "late-time" evolution. Both requirements (large number of cells per time step, and large total time evolution) can be quite challenging even for modern supercomputer systems.

This work is an extension of the presentations we have reported earlier, with the total time evolution of the RMHD jet increased by a factor of two, or roughly 250,000 cpu hours of computer time and a total of 50 terabytes of data. This extends the previous work from 125,000 cpu hours and 25 terabytes of data, and provides a substantial improvement in our confidence in the stability of the solvers used in the simulation. 


\subsection{Energy Loss, Energy Deposition Rate, and Momentum Transfer from Plasma Processes}

Hydrodynamic (HD), magneto-hydrodynamic (MHD) and even (RMHD) simulations are powerful tools for the investigation of physical phenomena in astrophysical settings, and have other important applications besides those in astrophysics.

However, such simulations neglect important aspects of the physics of astrophysical jets (in our particular examples): the microscopic interactions that occur because of the effects of particleparticle interactions and the interactions of particles with the collective effects that accompany a fully or partially ionized ambient medium (i.e., a plasma). This is a similar problem to that presented by the estimation of viscosity in hydrodynamic simulations.

In plasma processes, The system of equations used to determine the normalized wave energy densities for plasma-related processes is very "stiff". Scott et al. (1980) estimated the equilibrium solution of such a system of equations for heating of clusters of galaxies, and Rose et al. (1984) and Beall (1990) showed dynamical solutions that confirmed the stability of the equilibrium solutions. Solving the system of equations yields a time-dependent set of normalized wave energies (i.e., the ratio of the wave energy divided by the thermal energy of the plasma) that are generated as a result of jets interaction with the ambient medium. These solutions can yield an energy deposition rate $(d E / d t)$, an energy deposition length $(d E / d x)$, and ultimately, a momentum transfer rate $(d p / d t) \sim\left(1 / v_{b}\right) *(d E / d t)$, where $v_{b}$ is the beam velocity. This $d p / d t$ can be used to estimate the effects of plasma processes on the hydrodynamic evolution of the jet. We intend to address these effects in a later work.

For this part of the analysis, we suppose that a portion of the jet is composed of relativistic particles of either $e^{ \pm}, p-e^{-}$, or more generally, a charge-neutral, hadron- $e^{-}$jet, with a significantly lower density than the ambient medium. The primary energy loss for such a jet is supposed to be via hydrodynamical processes. Kundt $(1987,1999)$ also discusses the propagation of electron-positron jets.

Van der Westhuizen, et al. (2019), and Beall et al. (2018) show a late-time appearance of a fully three-dimensional simulation of an astrophysical jet's gas density, temperature, and pressure structure as seen in an $\mathrm{X}-\mathrm{Z}$ cross-section with a bulk velocity of $v=0.80 c$. Van der Westhuizen, et al., 2019, also show the $10 \mathrm{GHz}$ radio emission In that work, along with various, large-scale simulations with $512^{3}$ grid cells. The simulation demonstrated well-developed Rayleigh-Taylor instabilities at the jet-ambient medium boundary, as well as evolved Kelvin-Helmholtz instabilities along the longitudinal axis of the jet. Internal shocks in the jet channel are also noticeable, even though these do not manifest themselves in the radio emission estimated in van der Westhuizen's work (op cit).

The simulation size presented in the aforementioned work was $\sim 64 \mathrm{kpc}$ on a side and showed the structures developed after $2 \times 10^{3}$ time steps (roughly $10^{4}$ years). In that simulation, the jet-to-ambient medium density ratio is $1 / 100$ and the ratio of jet input pressure to ambient medium pressure is $5 \times 10^{-3}$ The simulation in that paper is based on a relativistic, hydrodynamic model, with estimation of the magnetic field intensity derived from pressure equilibrium with the ambient medium. Thus, the the calculation of the synchrotron emission in that paper is based on postprocessing of the estimated synchrotron emission from the jet particles and the ambient magnetic field in each element of the emitting region. 
Figure 4 of our paper (van der Westhuizen et al., 2019) shows an interesting comparison of the RHD jet density for four scenarios, and most importantly, the estimated synchrotron emissivity for the jet moving in the plane of the sky and perpendicular to the observer. It should be noted that there is a markedly different pattern of the jet number density, jet pressure, and temperature, and the synchrotron emissivity, both toward the head of the jet, and sometimes toward the jet base, depending on the specific aspects of the simulation.

The simulation we present here is also $512^{3}$ grid cells, but with a fully relativistic magnetohydrodynamic simulation, and with a significantly greater duration of the previously presented simulation (Beall et al., 2018) by a factor of two. The magnetic field in the jet has a poloidal/toroidal structure with both intensities of $1.0 \times 10^{1}$ Gauss, $\gamma=10$, and an ambient medium-to-jet density ration of 100/0.1. As can be clearly seen, the simulation shows well-developed Rayleigh-Taylor instabilities and significant shock structures along the jet core. In this simulation, we have used a PPM reconstruction and Runge-Kutta 3 algorithm, as well as the "tvdlf" solver from the PLUTO code (Mignone et al., in order to achieve a simulation resolution nearly as precise as the much larger and more computationally intensive $1024^{3}$ simulation.

Rose et al. (2002), and Beall et al. (2010) have compared the results of a PIC code simulations of an electron-positron jet propagating through an ambient medium of an electron-proton plasma with the solutions obtained by the wave population model code, and have found good agreement between the two results. At the same time, these papers demonstrate that the ambient medium is heated and partially entrained into the jet. The analysis also shows that a relativistic, low-density jet can interpenetrate an ambient gas or plasma.

An analytical calculation of the boost in energy of the electrons in the ambient medium to produce such a high energy tail, with $E_{\text {het }} \sim 30-100 k T$, is confirmed by PIC code simulations. Aside from altering the Landau damping rate (Rose et al., 2005), such a high-energy tail can greatly enhance line radiation over that expected for a thermal equilibrium calculation.

As part of our research into the micro-physics of the interaction of jets with an ambient medium, we continue to investigate the transfer of momentum from the jet, and expect to present these results shortly. In order to proceed to a more detailed analysis of the issue of momentum transfer, we have used modern PIC code simulations to study the dynamics of caviton formation, and have confirmed the work of Robinson and Newman (1990) in terms of the caviton's formation, evolution, and collapse.

\subsection{Results of Hydrodynamical Calculations}

As noted in the figure captions, Figure 1 shows an $\mathrm{x}-\mathrm{z}$ cut of a fully 3-D simulation of a relativistic jet in the magneto-hydrodynamic (RMHD) regime. This image we present here shows the density structure of a fully relativistic, magneto-hydrodynamic simulation of the latetime evolution of an astrophysical jet, using $512^{3}$ grid cells. The magnetic field in the jet has a poloidal/toroidal structure with both intensities of $1.0 \times 10^{1}$ Gauss, a ration of the jet energy vs. restmass, $\gamma=10$, and an ambient medium to jet density ration of 100/0.1. At this stage, the simulation shows well-developed Rayleigh-Taylor instabilities and significant shock structures along the jet core and where the jet interacts with the ambient medium. In this simulation, we have used a PPM reconstruction and Runge-Kutta 3 steps, as well as the "tvdlf" solver from the PLUTO code. 


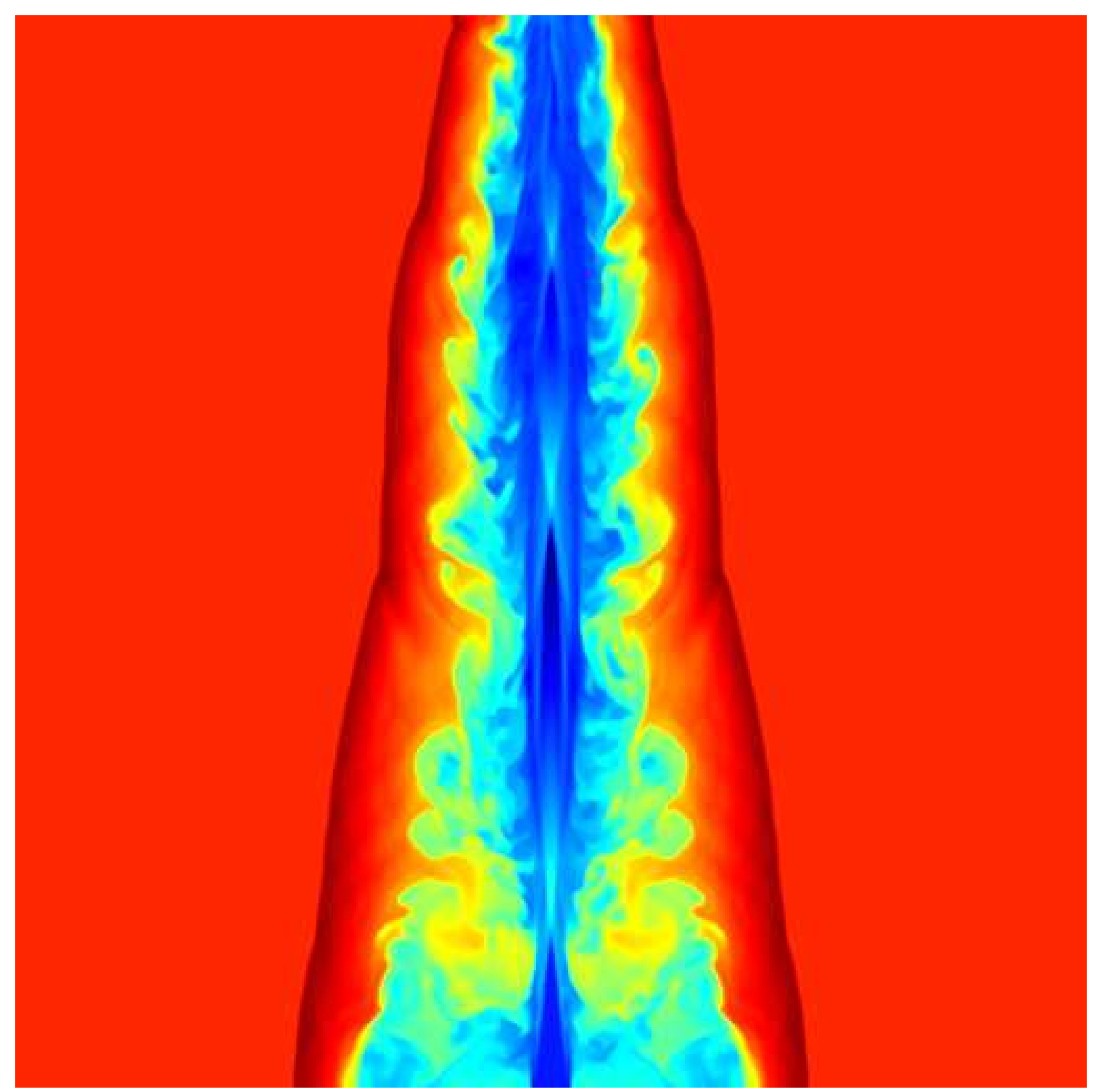

Figure 1: The image we present here shows the density structure of a fully relativistic, magneto-hydrodynamic simulation of the late-time evolution of an astrophysical jet, using $512^{3}$ grid cells. The magnetic field in the jet has a poloidal/toroidal structure with both intensities of $1.0 \times 10^{1}$ Gauss, a ration of the jet energy vs. rest-mass, $\gamma=10$, and an ambient medium to jet density ration of 100/0.1. At this stage, the simulation shows well-developed Rayleigh-Taylor instabilities and significant shock structures along the jet core and where the jet interacts with the ambient medium. In this simulation, we have used a PPM reconstruction and Runge-Kutta 3 steps, as well as the "tvdlf" solver from the PLUTO code. 


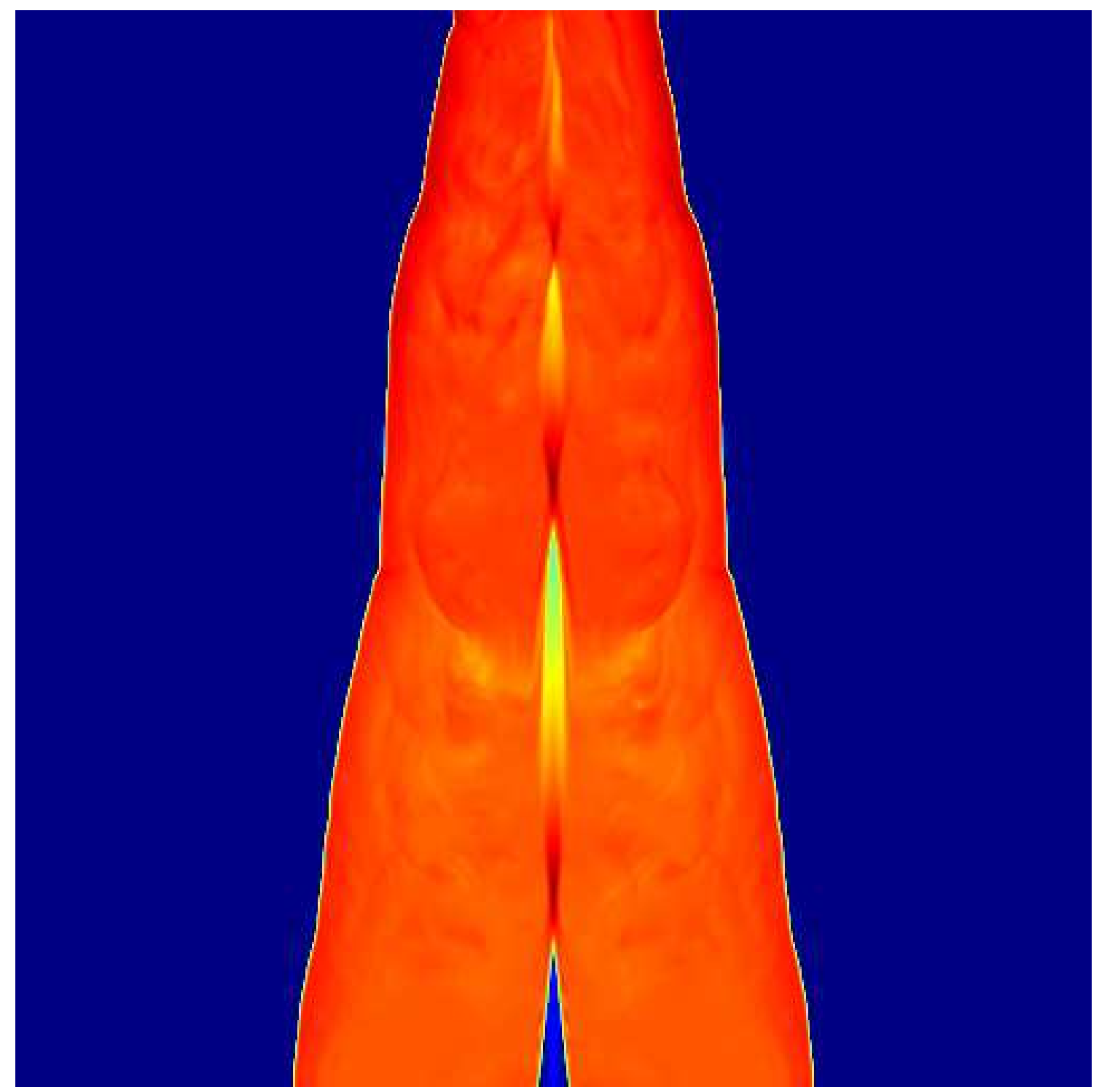

Figure 2: The image we present here shows the pressure structure of a fully relativistic, magnetohydrodynamic simulation of the late-time evolution of an astrophysical jet, with parameters identical to those in the previous figure, and with the identical configuration of "solvers" and time-steps for the PLUTO code.

Figures 2 and 3 show the same simulation at the same stage of the jet evolution for jet pressure and temperature structures, respectively.

The simulation shown in Figures 1 through 3 was done using the PLUTO code (Mignone et al. 2007) with our latest PPM and Runga-Kutta 3 time step runs, which yield more precise simulations of the jet evolution. The simulation volume in these images is $512^{3}$ simulation volume. The simulations are conducted using the Naval Research Laboratory's SGI Altix machine, POLAR. Our modifications to the graphical outputs provides $\mathrm{x}-\mathrm{z}$ slices at certain time steps in order to produce a convenient graphical guide. We use these images as"quick look" guides to investigate the details 


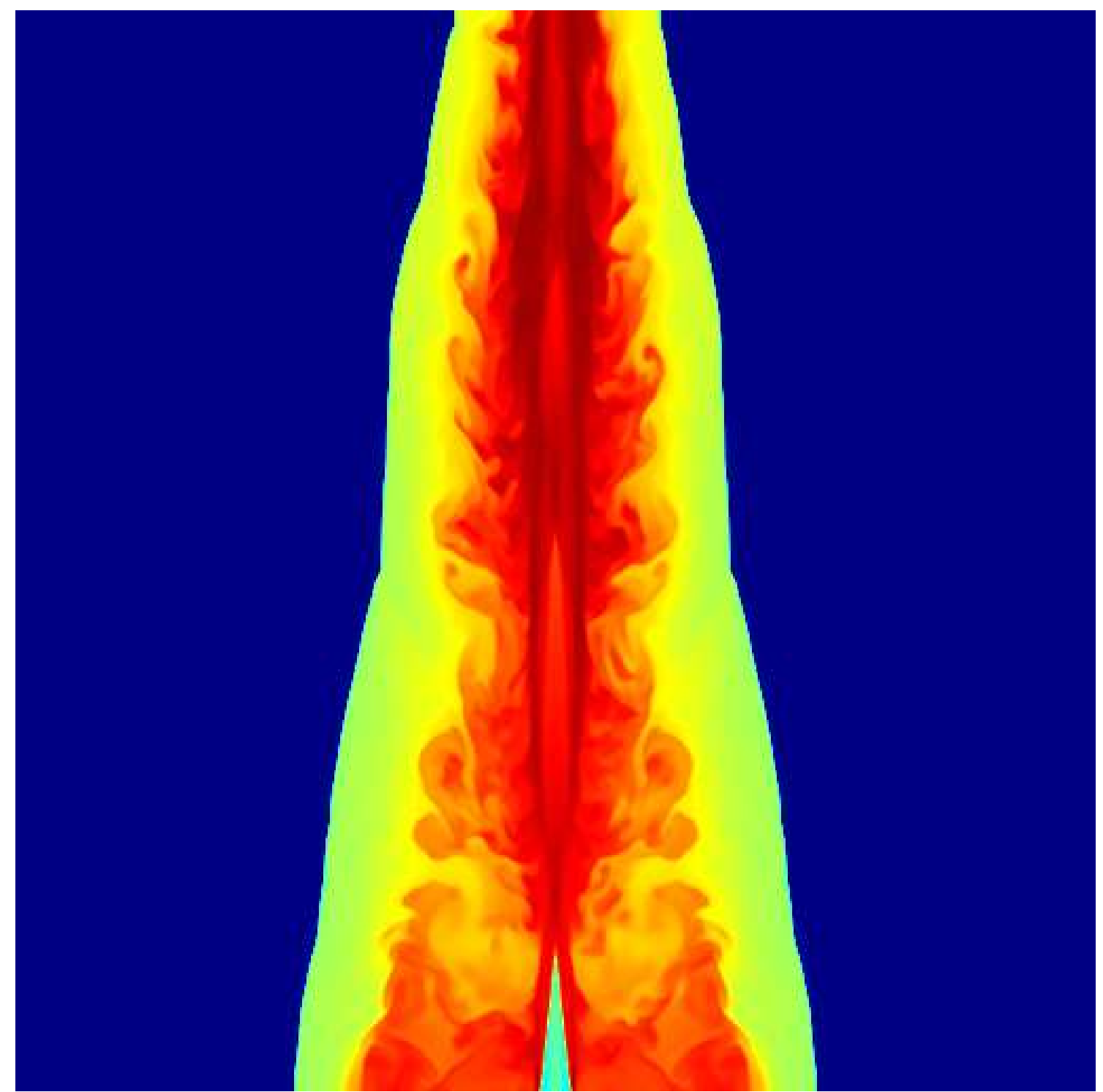

Figure 3: The image we present here shows the temperature structure of a fully relativistic, magnetohydrodynamic simulation of the late-time evolution of an astrophysical jet, with parameters identical to those in the previous figure, and with the identical configuration of "solvers" and time-steps for the PLUTO code.

of the simulations. In addition, in past simulations we have used the "external force" option in the code to simulate a disk gravity attraction that would illustrate coupling of the jet source to the jet-entrained ambient material.

Figure 5 shows a a comparison of the density structure of an astrophysical jet typical of our simulations, and its relation to the $15 \mathrm{GHz}$ radio light produced by our post-processing algorithms. Note the intense shock structure at the jet head. For this simulation, we have used a 512x64x64 and an relativistic hydrodynamic simulation and estimated the ambient magnetic field to be in pressure equilibrium with the jet density.

Figure 6 shows an image of the late-time jet simulation we have used in part to study the 


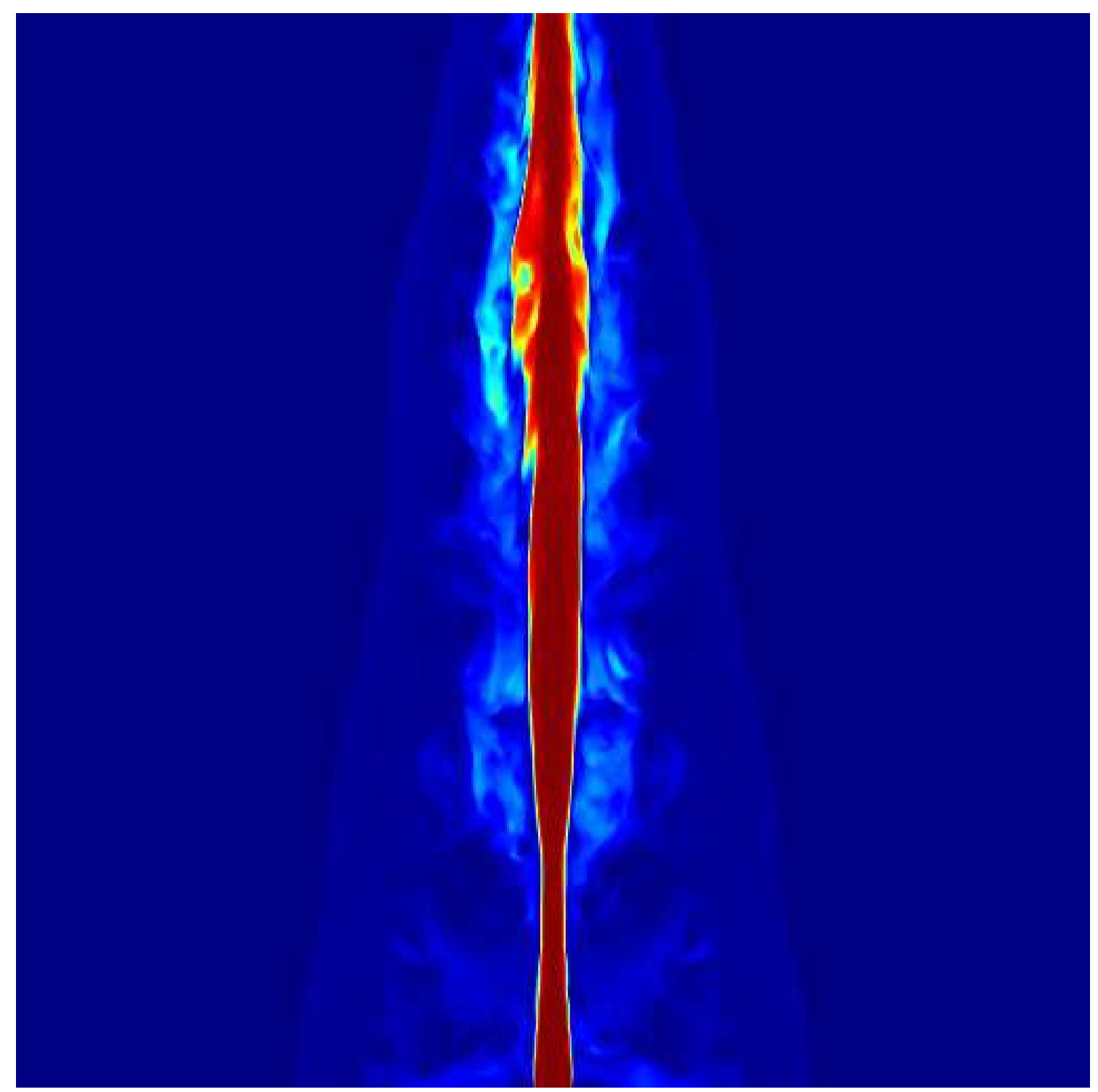

Figure 4: Figure 4 shows the velocity structure of the jet previously illustrated. From a perusal of Figures 1 through 4, it is possible to develop an intuition of the synchrotron emission from the jet column, but it is not a trivial enterprise to do so. We intend to show this in detail using the post-processing algorithms as illustrated in Figure 5.

synchrotron emission of a relativistic hydrodynamic jet that can be present in AGNs.

We are continuing to explore the parameter space of these jets by varying the bulk jet velocity, $\gamma$, and other jet parameters. Given certain simulation parameters, including a sufficient overburden of material in the ambient medium and a low-velocity jet, it is possible to demonstrate that the jet-propagation can be suppressed. This "frustrated jet" effect might explain the relatively truncated jets associated of jets with Seyfert galaxies. These effects have been discussed in some detail in Beall et al. (2015) 


\section{EDGE ON EMISSION, $\Phi=90^{\circ}$}
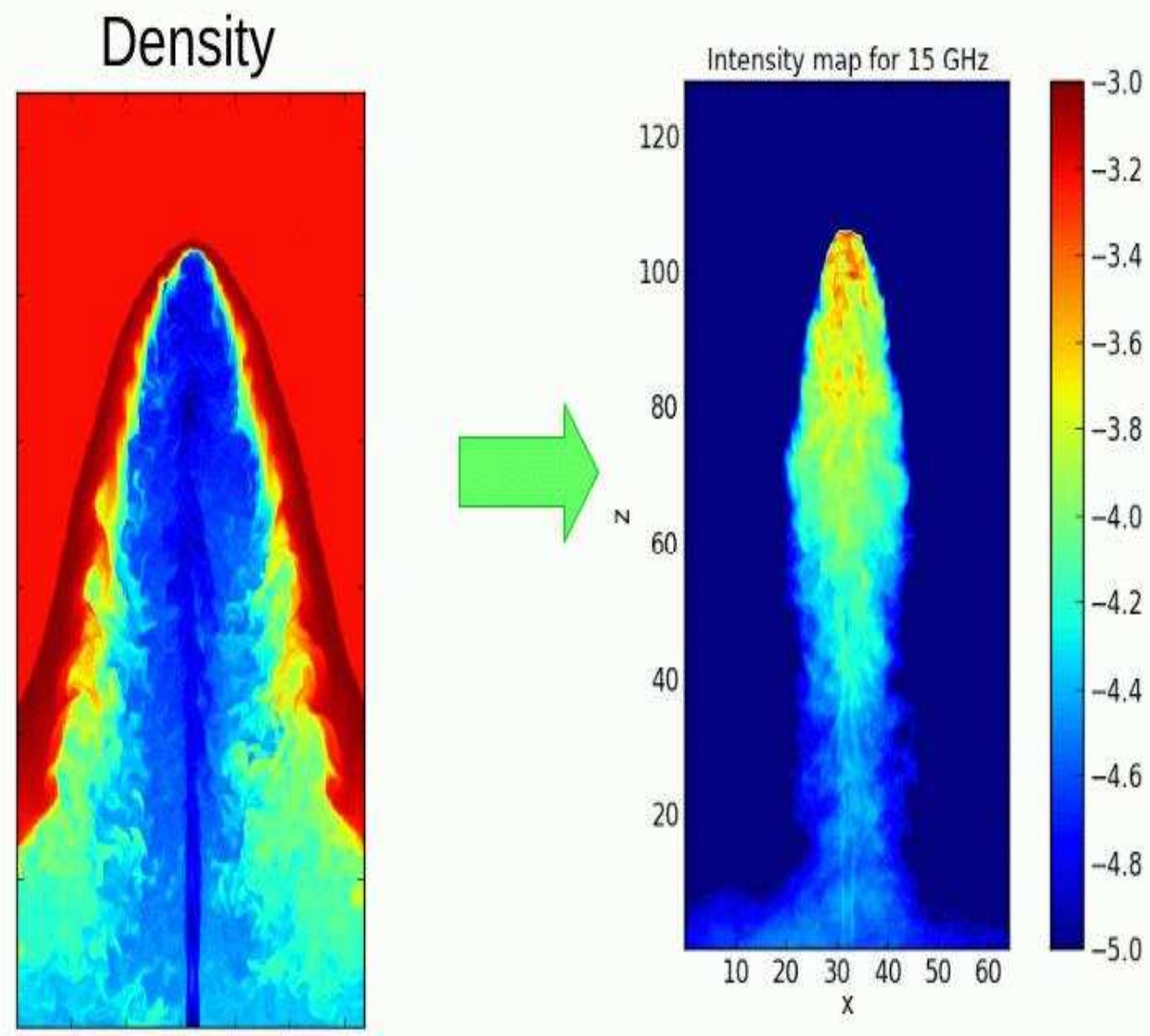

Figure 5: Figure 5 shows a comparison of the density structure of an astrophysical jet typical of the ones in our simulations and its relation to the $15 \mathrm{GHz}$ radio light produced by our post-processing from our 2019 paper. Note the intense shock structure at the jet head. For this simulation, we have used a 512x64x64 and an relativistic hydrodynamic simulation, and estimated the ambient magnetic field to be in pressure equilibrium with the jet density. 


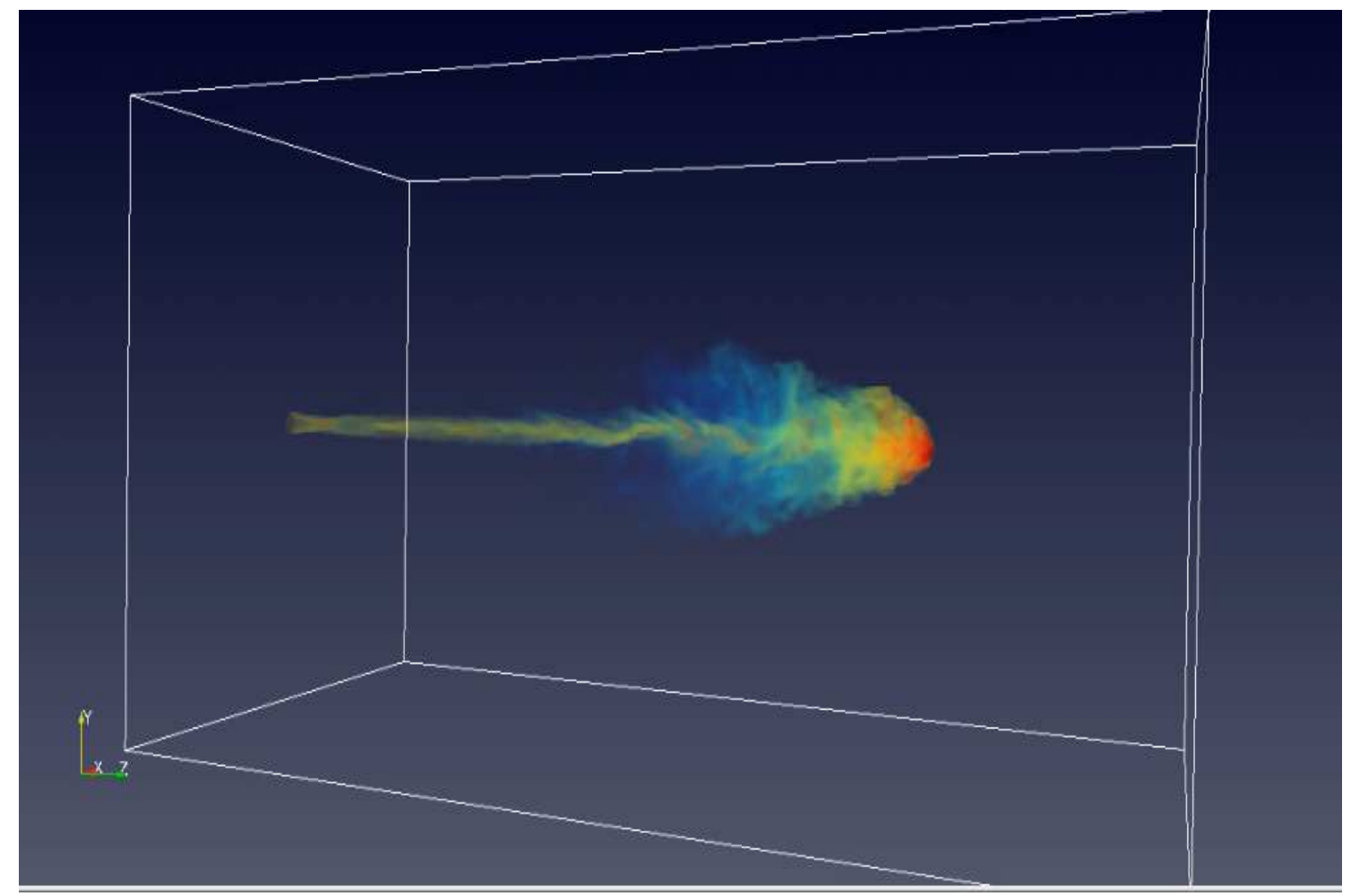

Figure 6: Figure 6 shows a typical jet from our simulations at an arbitrary angle to the observer, and near to its limit of propagation in uniform ambient medium. From this perspective, one can clearly see the radiobright cap on the jet head, the relatively quiescent jet column, and the development of both Kelvin-Helmholtz instabilities along the jet axis and the Rayleigh-Taylor instabilities developed near the jet cap.

\section{Concluding Remarks}

The effects of collective and particle processes, including plasma effects, can have observational consequences. Beall (1990) has noted that plasma processes can slow the jets rapidly, and Beall and Bednarek (1999) have shown that these effects can truncate the low-energy portion of the $\gamma$-rays spectrum (see their Figure 3 of Beall and Bednarek, 1999).

A similar effect will occur for particle-particle productions of neutrinos, pions, and (perhaps) neutrons (Atoyan and Dermer 2003). This could also reduce the expected neutrino flux from AGN. The presence of plasma processes in jets can also greatly enhance line radiation by generating high-energy tails on the Maxwell-Boltzmann distribution of the ambient medium, thus abrogating the assumption of thermal equilibrium.

JHB and MTW gratefully acknowledge support from the NASA Gamma-ray Astrophysics Program, the support of the Chief of Naval Research, and the Department of Defense High Performance Computing Modernization Program (HPCMP) 


\section{References}

[1] A. M. Atoyan and C. D. Dermer, 2003, Ap. J. 586, 79.

[2] Basson, J. F., and Alexander, P., 2002, The long-term effect of radio sources on the intracluster medium, MNRAS, 339, 353.

[3] Beall, J. H. and Bednarek, W., 1999, On the hadronic beam model for gamma-ray production in blazars, Ap.J., 510, 188.

[4] Beall J. H., 1990, Energy loss mechanisms for fast particles, Physical Processes in Hot Cosmic Plasmas, W. Brinkmann, A. C. Fabian, and F. Giovannelli, eds. (Kluwer Academic, London), p. 341.

[5] Beall, J.H., Rose, D.V., Wolff, M.T., and Lind, Kevin, 2015, "Hydrodynamic Simulations of Astrophysical Jets", Proceedings of Science (SISSA), 246, 061.

[6] Beall, J.H., 2016, "Astrophysical Jets: A Review", Proceedings of Science (SISSA), 269, 053.

[7] Beall, J.H., Rose, D.V., Lind, Kevin, Wolff, M.T., Brian van Soelen, Izak van der Westhuizen, and Pieter Meintjes, 2016, Large Scale Simulations of Astrophysical Jets, Proceedings of Science (SISSA), 269, 063

[8] Beall, J.H., 2017, "Astrophysical Jets: A Review", Proceedings of Science (SISSA), 306, 062,

[9] Beall, J.H., Rose, D.V., Lind, Kevin, Wolff, M.T., Brian van Soelen, Izak van der Westhuizen, and Pieter Meintjes, 2017, Large Scale Simulations of Astrophysical Jets, Proceedings of Science (SISSA), 306, 063.

[10] V.S. Belyaev, G.S. Bisnovatyi-Kogan, A.I. Gromov, B.V. Zagreev, A.V. Lobanov, A.P. Matafonov, S.G. Moiseenko, O.D. ToropinaNumerical Simulations of Magnetized Astrophysical Jets and Comparison with Laboratory Laser Experiments, 2019, arXiv.org > astro-ph > arXiv:1903.04199

[11] Colella, P., and Woodward, P. R., 1984, The piecewise parabolic method (PPM) for gasdynamical simulations, J. Comp. Phys., 54, 174.

[12] Fomalont, E. B., Geldzahler, B. J., and Bradshaw, C. F., 2001, Scorpius X-1: the evolution and nature of the twin compact radio lobes, Ap.J., 558, 283.

[13] Kaplan, S. A., and Tsytovich, V. N., 1973 Plasma Astrophysics, (Pergamon Press, Oxford).

[14] Krause, M., and Camenzind, M., 2003, Parameters for very light jets of cD galaxies, New Astron. Rev., 47, 573.

[15] Kundt, W., 1987, The sources and their models, in Astrophysical Jets and Their Engines, ed. W. Kundt (Reidel, Dordrecht, Netherlands), p. 1.

[16] Kundt, W. 1999, SS 433, Mem. S. A. It., 70, 1097. 
[17] Lister, M. L., Aller, H. D., Aller, H. F., Cohen, M. H., Homan, D. C., Kadler, M., Kellermann, K. I., Kovalev, Y. Y., Ros, E., Savolainen, T., Zensus, J. A., and Vermeulen, R. C., 2009, Astronom. J., 137, 3718.

[18] Mignone, A., Bodo, G., Massaglia, S., Matsakos, T., Tesileanu, O., Zanni, C., and Ferrari, A., 2007, Ap.J. Suppl., 170, 228.

[19] Mukherjee, D. Bicknell, Geoffrey V., Wagner, Alexander Y., Silk, J., Skutherland, R., 2018, Relativistic jet feedback III: feedback on gas disks, Monthly Notices of the Royal Astronomical Society, Volume 479, Issue 4, October 2018, Pages 5544-5566,

[20] Perucho. M., Martí, J.M., Cela, J.M., Hanasz, M., de La Cruz, R., Rubio, F., 2010, Stability of three-dimensional relativistic jets: implications for jet collimation, AA 519, A41

[21] Perucho, M., 2012, Jets in high-mass microquasars, Mem. S. A. It., 83, 297.

[22] Rose, W.K., Guillory, J., Beall, J.H., and Kainer, S., 1984, The interaction of relativistic charged-particle beams with interstellar clouds, Ap.J., 280, 550.

[23] Rose, W.K., Beall, J.H., Guillory, J., and Kainer, S., 1987, Radiation from relativistic beams interacting with interstellar gas, Ap.J., 314, 95.

[24] Rose, D.V., Guillory, J.U., and Beall, J.H., 2002, Comparison of particle-in-cell simulations and a wave-population model of electron-beam-plasma interactions, Phys. Plasmas, 9, Issue 3, 1000-1009.

[25] Rose, D. V., Guillory, J., and Beall, J. H., 2005, Enhanced Landau damping of finite amplitude electrostatic waves in the presence of suprathermal electron tails, Phys. Plasmas, 12, Issue 1, article id. 014501, 4 pp.

[26] Saxton, C. J., Bicknell, G. V., Sutherland, R. S., and Midgley S., 2005, Interactions of jets with inhomogeneous cloudy media, MNRAS, 359, 781.

[27] Scott, J. H., Holman, G. D., Ionson, J. A., and Papadopoulos, K., 1980, The heating of gas in clusters of galaxies by relativistic electrons - collective effects, Ap.J., 239, 769.

[28] Su, M., and Finkbeiner, D. P., 2012, Evidence for gamma-ray jets in the Milky Way, Ap.J., 753,61 .

[29] van der Westhuizen, I.P., ban Soelen, B., Meintjes, P.J., and Beall, J.H., MNRAS, Vol. 485, Issue 4, June 2019, 4658-4666.

[30] Zanni, C., Murante, G., Bodo, G., Massaglia, S., Rossi, P., Ferrari, A., 2005, Heating groups and clusters of galaxies: the role of AGN jets, Astron. Astrophys., 429, 399.

\section{DISCUSSION}

S. MOISENKO: What was the magnetization of the jet matter at the jet launching moment? 
JIM BEALL: The relativistic, magnetohydrodynamic simulations (RMHD) start with both a poloidal (along the jet axis) and a toroidal (like a donut) around the jet launch site. The strength of the magnetic fields in that scenario if specified as a fraction of the thermal energy in the ambient medium. But this quickly evolves as the magnetic field becomes more complex due to the interactions with the ambient material into which the jet propagates.

DIMITRI SALONIKA: Could you kindly describe the turbulence mentioned in your description of the jet simulations?

JIM BEALL: The turbulence is a consequence of the natural evolution of what is essentially a system of coupled differential equations driven in a highly non-linear mode. The simulation is of course a relativistic hydrodynamic or relativistic magneto-hydrodynamic one, depending on the particular run. But it behaves in the same manner as the chaotic, coupled systems because it essentially is one.

MARTIN TOPINKA: What if you wobble the source input angle a bit as in a precessing jet injection? Would that change much?

JIM BEALL: It would mimic some of what we actually see in the sky, as in the source 3C454.3. It is an interesting suggestion for our modeling . 